\title{
A Study on f Number of Points for Number of Points with Inter- Distance of Less Than, or for Number of Points with Inter-Distance of or More to Necessarily Exist on Plane \\ Taek SeungKim, Seung YoonBaek ${ }^{2}$ \\ ${ }^{1,2}$ Seoul Science High School, Seoul, Korea
}

\begin{abstract}
We defined $K B(\alpha, \beta, x, y)$ number of points with an inter-distance of $\beta$ or more to necessarily exist on a plane. Furthermore, we aimed to reduce the range of this minimum value. We first showed that the upper bound of this value could be scaled byxy, and further reduced the constant that was multiplied. We compared the upper bound of $K B(\alpha, \beta, x, y)$ and the Ramsey number in a special case and confirmed that $K B(\alpha, \beta, x, y)$ was a better upper bound than $R(x, y)$ except when $x, y$ were both small or trivial.
\end{abstract}

\section{Introduction}

Combinatorial geometry means solving problems using combinatorial ideas such as the pigeonhole principle from restricted geometric properties of arbitrary sets of points. The most basic conceivable constraint in combinatorial geometry is concerned with the distance between points, and related problems have been featured in various math competitions and magazines between the 1950s and 2010s. To determine the set of points satisfying given conditions, the pigeonhole principle or graph theory are commonly used. Considering the most general situation in combinatorial geometry, we intend to provide a generalization for distance restrictions. Among the related issues, we aim to investigate how many points should be given for $x$ number of points with an inter-distance of less than $\alpha$, or for $y$ number of points with an inter-distance of ßor more, to necessarily exist given arbitrary points on a plane. As a result, we aim to obtain the results for more effective number of points than those of previously described approaches, and further obtain optimal results for this study. This study will lead to further developments in the field of combinatorial geometry in the future.

\section{Definition of $K B(\alpha, \beta, x, y)$}

Definition For two positive real numbers $\alpha$, and two natural numbers $x$, the minimum natural number satisfying the following is defined as $K B(\alpha, \beta, x$,

No matter how distributed number of points are on a plane, there always exist number of points with an interdistance of less than, or number of points with an inter-distance of $\quad$, or more $\rfloor$. 


\section{Obtaining upper bound of $K B(\alpha, \beta, x, y)$}

\subsection{Generalization process}

To obtain the upper bound of $K B(\alpha, \beta, x$, we set the two lemmas described below.

\subsubsection{First lemma - upper bound of $K B(\alpha, \beta, x, y)$}

Lemmc. For a positive real number aand two natural numbers $\quad x$,

$$
K B(\alpha, \beta, x, y) \leq 3(y-1)(x-2)+1 \leq 3 x y
$$

Let $n=3(y-1)(x-2)+$. It is sufficient to show that for number of points on a plane, there exist number of points with an inter-distance of less than, or number of points with an inter-distance of or more.

[Proof by contradiction] Let us assume that number of points on the plane do not satisfy the above condition. Let us draw all the straight lines that can be made with any two of these points. The number of straight lines drawn is at most $n^{\prime}$.

Let be a certain straight line that is not parallel to these straight lines and draw a-rectangular coordinate system in which is used as -axis.

Since the -coordinates of the given points are different, let $P_{1}, P_{2}, \ldots$ from the smallest $p_{\text {-coordinate. Let us }}$ define the natural number and , and a set $C_{k}(k=1,2, \ldots, r$

as inductively for :

(ㄱ) , $i_{1}=1, C_{1}=\mathrm{e} \mid 0 \leq \overline{P_{1} P_{e}}<$

(L)When natural numbers $i_{1}, i_{2}, \ldots$, and sets $C_{1}, C_{2}, \ldots$, are defined,

$$
\begin{aligned}
& \text { - if } \\
& \bigcup_{j=1}^{k} C_{j} \neq\{1,2, \ldots, n\} \text { then } \mathrm{i}_{\mathrm{k}+1}=\min \left\{\epsilon \mid \epsilon \notin \bigcup_{j=1}^{k} C_{j}\right\}, \mathrm{C}_{-}\{\mathrm{k}+1\}=\left\{\epsilon \mid 0 \leq \overline{\mathrm{P}_{\mathrm{k}+1} \mathrm{P}_{\epsilon}}<\alpha\right\} \\
& \text { - if } \mathrm{U}_{j=1}^{k} C_{j} \neq 1,2, \ldots, \text {, then } \quad \text { is defined as . }
\end{aligned}
$$

First, if $1 \leq a<b \leq$, then, $P_{i_{k}} \notin\left(\right.$ and thus, $\overline{P_{i_{r}} P_{i_{k}}} \geq$. Therefore, $P_{i_{1}}, P_{i_{2}}, \ldots . F$ are number of points with an inter-distance of $\quad$ or more; and from the assumption by contradiction, $\quad m \leq y-$ 
Furthermore, by the definition of $m P_{i_{1}}, P_{i_{2}}, \ldots . P_{n} \in U_{j=1}^{m}$. Hence,

because $n>3(y-1)(x-2)=(y-1)(3 x-$ and $\quad m \leq y-$, by the pigeonhole principle, there exists some that includes $3 x$-or more number of . Let $\epsilon_{0}<\epsilon_{1}<\cdots<\epsilon_{3 x}$ by choosing $3 x$-number of from the smallest, where $P_{e} \in$ (by definition of, $i_{j}, i_{j}=$ )Here, we can see that $3 x-$ number of points such as $P_{e_{1}}, P_{e_{2}}, \ldots \ldots, P_{e_{3 x}}$ are located in the region of the semicircle where -coordinates are larger than $\quad$ inside the circle, whose radius is with its center at $\quad F$.

Fig. 3.1.1

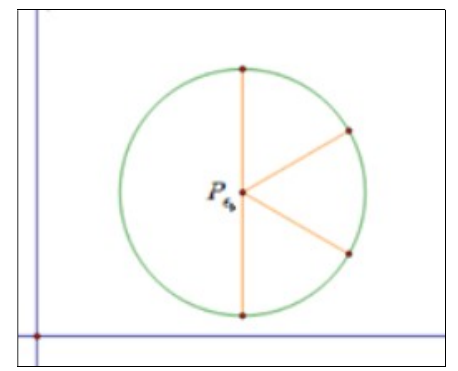

This region is divided into three sectoral regions with central angle of 6, as shown in Fig. 3.1.1. (The boundaries between the regions are arbitrarily included.) By the pigeonhole principle, there exist $\quad x$ - number of points in the same region among the points of $\quad P_{e_{1}}, P_{e_{2}}, \ldots \ldots, P_{e_{3 x}}$ such that $3 x-5=3(x-2)+$.

The number of points including these points and $f$ have an inter-distance of less than , which is contradictory to the assumption. Thus, the assumption by contradiction is false, and the given proposition is proven as valid.

\subsubsection{Second lemma}

LemmaFor any natural number and positive real number , if there exist $\quad 7 k$ tpoints on a plane which have an inter-distance of $\quad$ or more, there must be $\quad k$ tpoints that have an inter-distance of $\sqrt{\text { ior more. }}$

Since the plane is a space that can be expanded or contracted, it is sufficient to prove this claim for $\alpha-$. Let us prove this claim using the mathematical induction for .

$$
\text { i) } k=1
$$

Let us call the given points $P_{1}, P_{2}, \ldots$. Let us take a rectangular coordinate system ( -axis, -axis) arbitrarily, and let 
the point having the smallest -coordinate be among the given points without loss of generality. As shown in Fig. 3.1.2.1, let us draw circles with radius of and , respectively, with as its center. Let $\left.C=P_{1} \mid 1 \leq \overline{P_{1} P_{i}}<\sqrt{3}\right\}$

Fig. 3.1.2.1

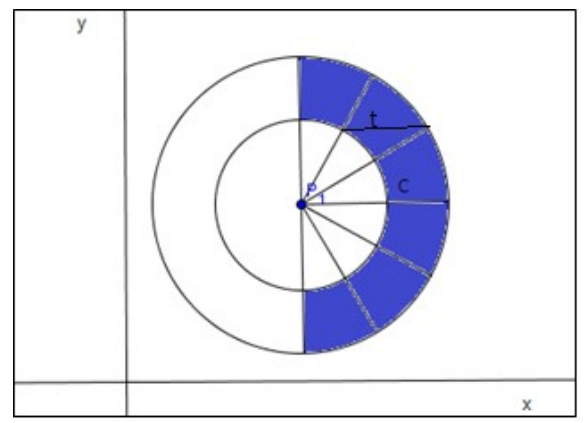

Any element in Fig. 3.1.2.1 is included in the region having a larger -coordinate than among the regions between two circles.(The "region" includes the inner circumference but excludes the outer circumference.)Now let us divide this region into six sections by drawing six circular sectors having as the central angle, as shown in Fig. 3.1.2.1. (However, the boundaries between the sections are arbitrarily included in either section.) For each section, the distance between any two points in the section is less than

Here, we can obtain $t^{2}=1^{2}+(\sqrt{3})^{2}-2 \cdot 1 \cdot \sqrt{3} \cdot \cos 30^{\circ}=$ by the Second Cosine Law.Thus, $\quad t=$ and the distance between any two points in the same section is less than 1 . Namely, there cannot be more than two given points in one section. Because any of the seven points other than may not be an element in , let that point be without loss of generality. Then, since $P_{2} \notin C, \overline{P_{1} P_{2}} \geq \sqrt{3}, P_{1}$, are two points satisfying the given condition, the above proposition holds true.

ii) If the claim is true when $\quad k=k_{0}-1\left(k_{0} \geq\right.$, then let us show the claim is also true when $k=$.

Let the set of given points be $S=\left\{P_{1}, P_{2}, \ldots \ldots P_{7 k_{0}}\right.$. Let us take a rectangular coordinate system ( -axis, -axis) arbitrarily, and let the point having the smallest -coordinate be among the given points without loss of generality. As shown inFig. 3.1.2.2, let us draw circles with radius of and , respectively, with as its center. Let $C=\left\{P_{i} \mid 1 \leq \overline{P_{1} P_{i}}<\sqrt{:}\right.$

Fig. 3.1.2.2 


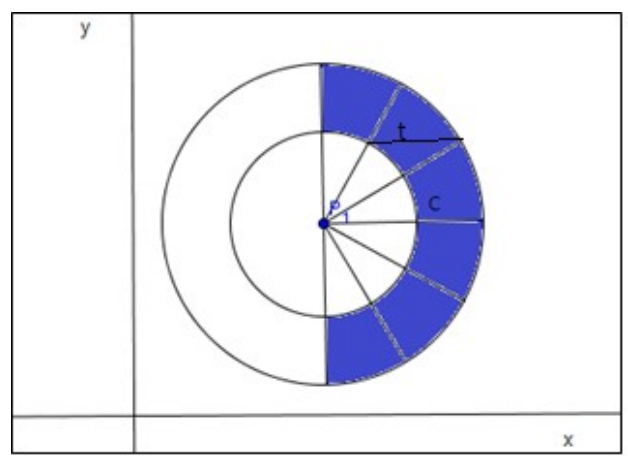

Any element in is included in the region having a larger -coordinate than among the regions between two circles. (The "region" includes the inner circumference but excludes the outer circumference.) Now let us divide this region into six sections by drawing six circular sectors with 3as the central angle, as shown in Fig. 3.1.2.2. (However, the boundaries between the sections are arbitrarily included in either section.)

Fig. 3.1.2.3

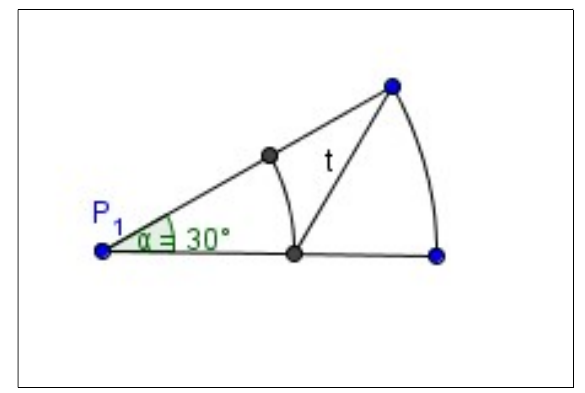

For each section, the distance between any two points in the section is less than Here, we can obtain $t^{2}=1^{2}+(\sqrt{3})^{2}-2 \times 1 \times \sqrt{3} . \cos 30^{\circ}=$ by the Second Cosine Law.Thus, $t=$ and the distance between any two points in the same section is less than 1 . Specifically, there cannot be more than two given points in one section. Hence, $\quad|C| \leq$ and there exist at least $\quad 7\left(k_{0}-1\right)+$ number of points at a distance of or more from . among the given points. Here, by induction assumption, it can be seen that thereexist number of points that have an inter-distance of, or more. In other words, these number of points and the $k_{0}+$ number of points including satisfy the condition. Thus, the above proposition also holds true for $\quad k=l$

Therefore, the above proposition holds true for any natural number .

\subsubsection{Upper bound of generalKB( $\alpha, \beta, x, y)$}

The following Theoremwas obtained from Lemma 1 and Lemmc. 
Theorem. For any positive real number , two natural numbers $x$, and non-negative integer ,

$K B\left(\alpha, \sqrt{3^{m}} \alpha, x, y\right) \leq 3 . \quad 7^{m}$

Let us prove that the claim holds true for by mathematical induction.

i) $\quad m=$ : True by Lemmc.

ii) If it holds true when $m-r$, then let us prove that it also holds true when $\quad\left(m_{0} \geq 0\right) m-m_{0}+$.

It is sufficient to show that among arbitrary $3 \times 7^{m_{0}+1}$ : number of points on a plane, there exist $\quad$ number of points that have an inter-distance of less than , or number of points that have an inter-distance of $\sqrt{3^{m_{0}+}}$ or more. Since $K B\left(\alpha, \sqrt{3^{m_{0}}} \alpha, x, 7 y\right) \leq 3 \times 7^{m_{0}+1}$. by induction assumption, there must be number of points that have an inter-distance of less than , or $\quad$ : number of points that have an inter-distance of $\sqrt{3^{m_{0}}+1}$ or more among these points.

When there exist $\quad:$ number of points that have an inter-distance of $\quad \sqrt{3^{m_{0}+1}}$ or more, there exist $\quad$ number of points that have an inter-distance of $\sqrt{3^{m_{0}+1}}$ or more among these points by Lemmc. Moreover,it holds true when there exist number of points that have an inter-distance of less than .Since the claim holds true for both cases, it is also true for $\quad m=m_{0}+$

Therefore, the claim holds true for all non-negative integer $m$.

\subsection{Obtaining sophisticated upper bound}

Theorem 3can be expressed as follows.

Theorem 3. For any two positive real numbers $\alpha, \beta$, and two natural numbers $x, y$, the following holds true.

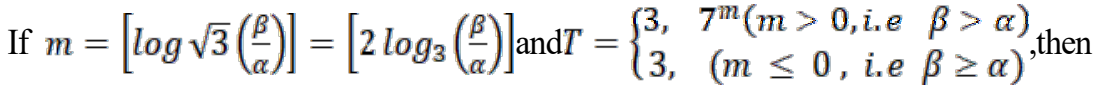

$$
\begin{aligned}
& K B(\alpha, \beta, x, y) \leq \text { Txy }
\end{aligned}
$$

Here, we set a new goal to reduce the $T$ value when $m>0$, i.e. $\beta>\alpha$.

\subsubsection{Variation of Lemma 2}

We decided to calculate the threshold value of Lemma 2 to find the minimum value of the value, when $\beta>$. In order to extend , into a general real number $r>$, and further use a similar proof method, the case $r<$ is 
primarily considered. In order to generalize the region $\quad A B$ (excluding arc

$\bar{F}$ and including arc $\bar{I}$ that appeared in the previous proof process as $\quad \overline{A Y}=$ shown in Fig. 3.2.1.1, the distance between any two points inside this region should be less than 1 . Thus, when $\overline{A B} \leq$, the same proof process can be applied. Let the central angle be Fig. 3.2.1.1

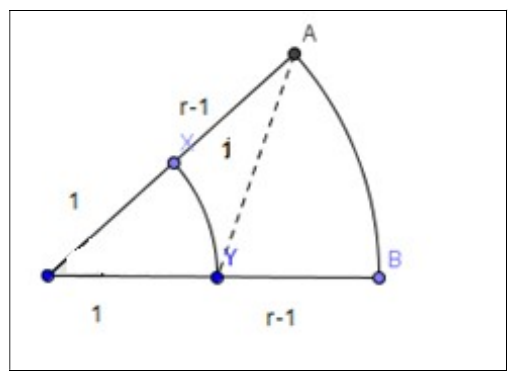

As shown in Fig. 3.2.1.1, since $\quad \cos \theta=\frac{r}{2}, 1-\sin ^{2} \frac{\theta}{2}=, \quad$ i.e., $\quad \sin \frac{\theta}{2}=\sqrt{\frac{1}{2}-}$.

Hence, $\overline{A B}=2 r \sin \frac{\theta}{2}=r \sqrt{2-r} \leq$, i.e., $\quad r^{3}-2 r^{2}+1 \geq$ can be obtained.Factorizing and rearranging this inequality lead to $\frac{1+\sqrt{5}}{2} \leq r<$ because $\quad r^{2}-r-1 \geq$ and $1<r<$.Here, we obtain Lemme.

Lemmc. If a positive real number is given, and for any real number $\quad r\left(\frac{1+\sqrt{5}}{2} \leq r<\right)$ $M=\left[\frac{\pi}{\cos ^{-1}(?}\right.$, the arbitrary $(M+1) k+$ number of points on a plane have an inter-distance of or more, and there must exist $\quad(k+$ number of points that have an inter-distance of $i$ or more.

Let us apply the same proof method to the case where $1<r<\frac{1+}{i}$

Fig. 3.2.1.2

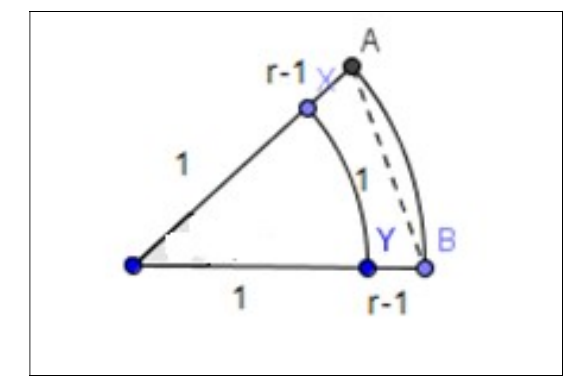

As shown in Fig. 3.2.1.2,let $\overline{A B}=$ to use the region $A B$ l(excluding arc $\bar{t}$ and including arc $\quad \overline{2}$ ) for proof; it is sufficient if $\quad \overline{A Y}=\bar{B} X \leq$. Let the central angel be ${ }^{\theta}$. As shown in Fig. 3.2.1.2, since 
$\sin \frac{\theta}{2}=\quad, \quad \cos \theta=1-\sin ^{2} \frac{\theta}{2}=1-$. Here, $\quad \overline{A Y^{2}}=1+r^{2}-2 r \cos \theta=1+r^{2}-2 r+\frac{1}{r} \leq$,

i.e., $r^{3}-2 r^{2}+1 \leq$, and factorizing and rearranging this inequality lead to $\quad(r-1)\left(r^{2}-r-1\right) \leq$ because

$1<r$ and $\quad 1<r \leq \frac{1+}{:}$. Hence, we can obtain Lemma

Lemmc. If a positive real number is given, and for any real number $\quad r\left(1<r<\frac{1+\sqrt{ }}{2}\right.$

$M=\left[\frac{\pi}{2 \sin ^{-1}\left(\frac{1}{21}\right.}\right.$, the arbitrary $\quad(M+1) k+$ number of points on a plane have an inter-distance of or more,

and there must exist $\quad(k+$ number of points that have an inter-distance of $\quad i$ or more.

$$
\theta=\left\{\begin{array}{c}
\cos ^{-1}\left(\frac{r}{2}\right),\left(\frac{1+\sqrt{5}}{2} \leq r<2\right) \\
\sin ^{-1}\left(\frac{1}{2 r}\right), \quad\left(1<r<\frac{1+\sqrt{5}}{2}\right), M=\lceil
\end{array}\right.
$$

Now let us prove that Lemma and Lemma hold true. This also can be proven as follows.

$\ulcorner$ For any real number $\quad r(1<r<$ and for $\mathrm{M}$ defined as above,the arbitrary $\quad(M+1) k+$ number of points on a plane have an inter-distance of $\quad$ or more, and there must exist $\quad(k+$ number of points that have an interdistance of $\quad i$ or more among the points. (When $\quad k=$, one point is regarded as satisfying the condition.) $\lrcorner$

$p$ Since the plane is a space that can be expanded or contracted, it is sufficient to prove this claim for $\alpha=$. Let us prove this claim using mathematical induction for .

i) $k=$ : It holds true.

ii)If the claim is true when $k=k_{0}-1\left(k_{0} \geq 1\right)$, let us show the claim is also true when $k=$ Let us call the given points as $S=\left\{P_{1}, P_{2}, \ldots, P_{(M+1)} k_{0}+\right.$. Let us take a rectangular coordinate system ( -axis, axis) arbitrarily, and let the point having the smallest -coordinate be among the given points without loss of generality. As shown in Fig. 3.1.2.3, let us draw circles with a radius of 1, and respectively, and as its center. Let $\quad C=P_{i} \mid 1 \leq \overline{P_{1} P_{i}}<$

Fig. 3.2.1.3 


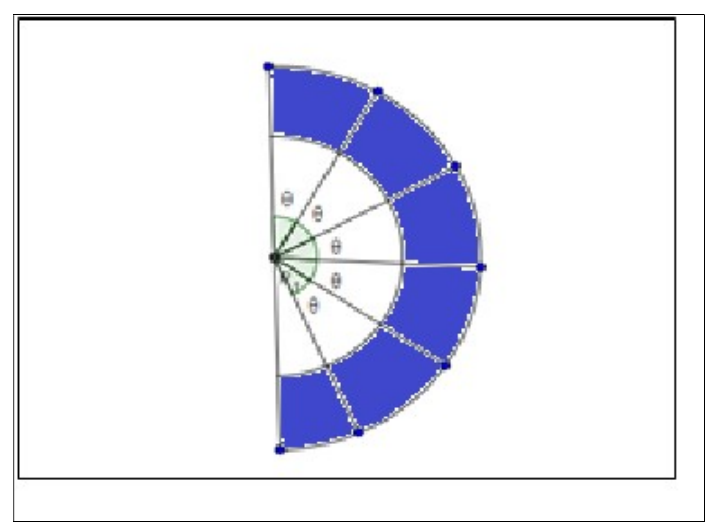

Any element in is included in the region having a larger -coordinate than among the regions between two circles. Now let us divide this region into sections by drawing circular sectors having or less as its central angle, as shown in Fig. 3.1.2.3. (However, the boundaries between the sections are arbitrarily included in either section.)

As previously proven, the distance between any two points in the same section is less than 1 . In other words, two or more given points in one section cannot exist. Hence, $\quad|C| \leq$, and there exist at least

$$
(M+1)\left(k_{0}-1\right)+\text { number of points having a distance of } \quad \text { or more from among the given points }
$$

Here, by induction assumption, there can exist number of points that have an inter-distance of $r$ or more. In other words, these number of points and the $k_{0}+$ number of points including satisfy the condition. Thus, the above proposition also holds true for $\quad k=$.

Therefore, the proposition holds true for any natural number .

\subsubsection{Theorem Refinement process}

Now let us find more sophisticated value to reduce the value when $\beta>$. Thus far, we have come up with Lemma and Lemma to obtain a solution to the case where the value is large through the following experiment.

"Experiment": Select number of points having an inter-distance of or more among the $(M+1$ number of points having an inter-distance of or more.

Since we need to perform trials that satisfy $r^{N}, \alpha \geq$ in this experiment, at least 
$N=\left[\log _{r}\left(\frac{\beta}{a}\right)\right] \log _{r}$ (trials should be performed. Since $\frac{\beta}{a}>, \quad \log _{r}\left(\frac{\beta}{\alpha}\right)>$ when $\quad$ number of points are initially given on a plane, an experiment with trials wasperformed, and we can select $\frac{X}{(M+1}$ number of points.

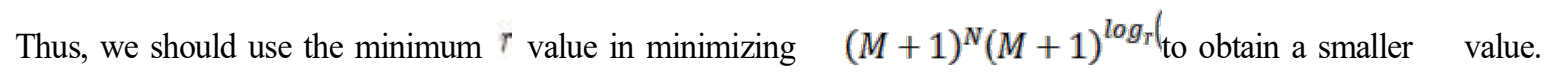
Since $(M+1)^{\log _{r}\left(\frac{\beta}{\alpha}\right)}=(M+1)^{\frac{m m}{\ln _{n}}}$ should be minimized, $\quad(M+1)^{\bar{n}}$ should be minimized, i.e., the value should be found by minimizing $\ln (M+1)^{\frac{1}{\ln (r)}}=\frac{\ln (M+1)}{\ln r} \log _{r}(M+$.

Graph 3.2.2

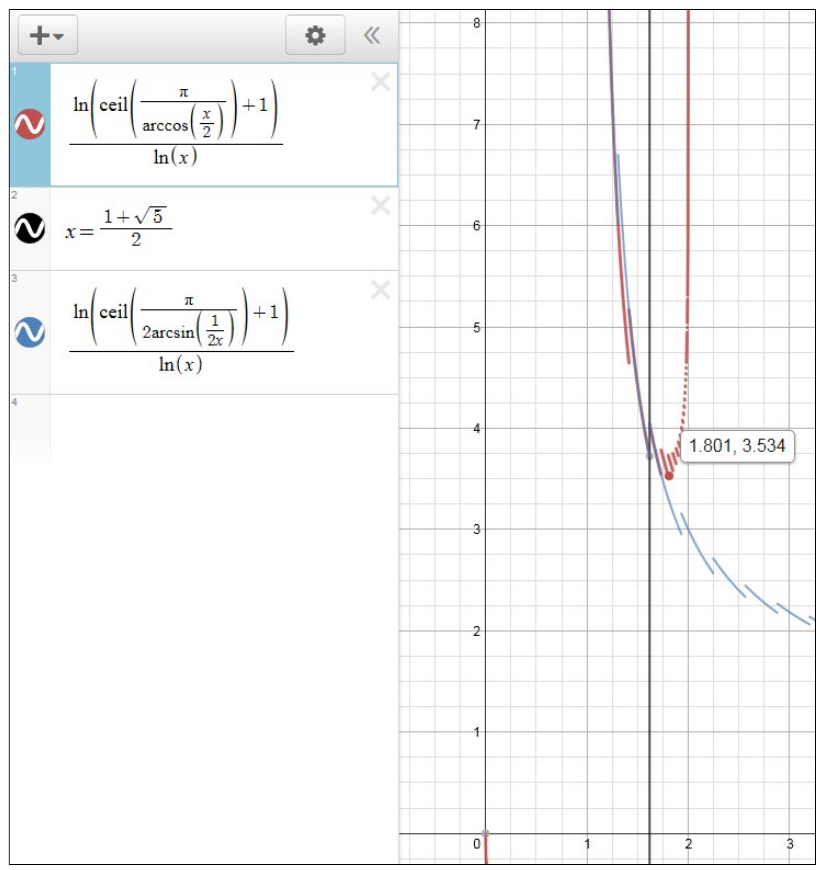

By finding the optimal value through Graph 3.2.2, then $r=2 \cos \frac{\pi}{7}=1.81$, where $M=7$. Thus, we can obtain a more sophisticated result, Theoren.

Theoren. For any real number $\quad \alpha, \beta$ >, and natural numbers $\quad x$, the following holds true.

If $m^{\prime}=\left[\log _{2 \cos \frac{\pi}{7}\left(\frac{\beta}{a},\right.} T=\left\{\begin{array}{l}3, S^{m}\left(m^{\prime}>0, \text { i. } e \beta>\text {, }\right. \\ 3, \quad\left(m^{\prime} \leq 0, \text { i. } \beta^{\prime}, \mathrm{c}\right.\end{array}\right.\right.$, then $\quad K B(\alpha, \beta, x, y) \leq T$ :

i.e., when $\quad \beta>$, we obtain a more effective as $T=3 \times s\left\lceil\log _{2 \cos \frac{\pi}{7}}\left(\frac{\beta}{a}\right.\right.$. 


\section{Relationship analysis between $K B(\alpha, \beta, x, y)$ and $R(x, y)$}

In order to confirm whether Theoreris a meaningful result, we sought to obtain a better result in comparison with a self-evident result in a special case.

When $\quad \alpha=\beta\left(m^{\prime}=0\right)$ (i.e.,Lemma, Theoreris statedas follows.

$\ulcorner$ For any positive real number and natural numbers $\quad x, \quad K B(\alpha, \alpha, x, y) \leq 3 .$.

In order to show that 3 : is a meaningful result, we compared this result with the known concept Ramsey number. For two natural numbers $\quad x$, a Ramsey number $\quad R(x$, is defined as follows.

For a given natural number , suppose that all line segments $\quad\left(\frac{n(n+1}{2}\right.$ of a complete graph with $n_{\text {number of }}$ points are filled with one of two colors, and . Let $n$ be a "good number" if there exist a complete graph with number of points filled with the color , or a complete graph with number of points filled with the color ,regardless of how it is painted. In this case, there must exist at least one "good number," and the minimum "good number" is defined as $R(x$,

Claim. For any positive real number and two natural numbers $\quad x, K B(\alpha, \alpha, x, y) \leq R(x$,

$p$ Suppose that $n=R(x$, it is sufficient to show that among arbitrary number of points on a plane,there exist number of points having an inter-distance of less than , or number of points having an inter-distance of or more. Let the given number of points be $P_{1}, P_{2}, \ldots$. Now, let us draw a complete graphwith $V_{1}, V_{2}, \ldots$ as its vertices to connect and with a red line if $P_{i}, P_{j} \geq$, and connect and with a blue line if $P_{i}, P_{j}<$ By the definition of $R(x$, there exists a red-colored complete graph with number of points or a blue-colored complete graph with number of points. In the first case, the exist number of points having an inter-distance of or more; in the second case, the exist number of points having an inter-distance of less than . Therefore, the proposition holds true.

$C_{1}$ where $\forall \mathrm{t} \in \mathrm{N}, \mathrm{R}(3, \mathrm{t}) \geq \mathrm{c} \frac{\mathrm{t}^{2}}{\log \mathrm{t}}$ 
In other words, the proposition still holds true if $R(x$, is applied instead of 3 .. Thus, when the numbers of and are large, where $R(x, y)>3, \quad$ Theoren, where $\alpha=\beta, m=$, i.e., $\quad$ Lemm, is meaningful.

According to known results, the following hold true:

- According to P. Erdös [1], there are suitable constants.

- According to Joel Spencer [2], the following holds.

- According to P. Erdös [1], there exista proper constant $C_{1}$ where $\forall t \in N, R(3, t) \geq c$;

- According to Joel Spencer [2], the following holds true.

$\forall k \in N, R(k, k) \geq \frac{k 2^{\frac{k}{2}}}{\epsilon \sqrt{2}+0}$,

- For a fixed $\quad k$, as $t \rightarrow \infty, R(k, t) \geq t^{\frac{k-1}{2}+0}$, holds true.

Therefore, the following cases have more accurate results than a Ramsey number if $\alpha=\beta, m^{\prime}=$ for $\quad$ Theoren.

a. If $3 \in\{x\}$ When is sufficiently large, it is true that $R(3, t) \geq c_{\overline{1}}$.

b. If or $4 \geq x \geq y \geq \frac{s^{\frac{x}{2}}}{3\{e \sqrt{2}+0\}}$, then it is true that

$$
\begin{array}{lll}
x \leq y \leq \frac{s^{\frac{x}{2}}}{3 e \sqrt{2}+0} & \Rightarrow & R(x, y) \geq R(x, x) \geq \frac{x^{2^{2}}}{e \sqrt{2}+0(1)} \geq 3 \text {, and } \\
y \leq x \leq \frac{2^{\frac{y}{2}}}{3 e \sqrt{2}+0} \quad \Rightarrow \quad R(x, y) \geq R(y, y) \geq \frac{y^{\frac{y}{2}}}{e \sqrt{2}+0(1)} \geq 3 .
\end{array}
$$

c.If $y \gg x \geq 4$ or $x \gg y \geq$, then it is true that

$$
\begin{array}{lll}
y \gg & \Rightarrow & R(x, y) \geq y^{\frac{x-1}{2}+0(1)} \geq y^{\frac{3}{2}} \geq 3 \text {., and } \\
x \gg & \Rightarrow \quad R(x, y) \geq x^{\frac{y-1}{2}+0(1)} \geq x^{\frac{3}{2}} \geq 3 .
\end{array}
$$

Here, when $x$ are sufficiently large, our theory has been shown to be practical in most cases, whether the difference is extremely large (c) or the difference is not significantly large (b).

Depending on $\quad x$, particularly when $\quad x$ are small, the conclusions drawn by $R(x$, could be more accurate than 
the case where $\quad \alpha=\beta, m^{\prime}=$ for $\quad$ Theorer. Table 4.1 below summarizes the known values or the known upper and lower bounds of the Ramsey number $\quad R(k$,when the values lare small. Here, all Ramsey numbers other than $R$ (5, satisfy $\quad R(k, l)<3$, and are still more effective than the case where $\quad \alpha=\beta, m^{\prime}=$ for $\quad$ Theoren.

\begin{tabular}{|c|c|c|c|c|c|}
\hline$k^{l}$ & 3 & 4 & 5 & 6 & 7 \\
\hline 3 & 6 & 9 & 14 & 18 & 23 \\
\hline 4 & & 18 & 25 & $\begin{array}{l}35 \\
41\end{array}$ & $\begin{array}{l}49 \\
61\end{array}$ \\
\hline 5 & & & $\begin{array}{l}43 \\
49 \\
\end{array}$ & $\begin{array}{l}58 \\
87 \\
\end{array}$ & $\begin{array}{r}80 \\
143 \\
\end{array}$ \\
\hline
\end{tabular}

Table 4.1: Known nontrivial values and bounds for Ramsey numbers $R(k, l)$

\section{Summary and suggestions}

$$
m=\left\lceil\log \sqrt{3}\left(\frac{\beta}{\alpha}\right) \mid=\left\lceil 2 \log _{3}\left(\frac{\beta}{\alpha}\right)\right\rceil\right.
$$

\subsection{Summary}

Starting from the basic case with the same distance $\quad\left(\alpha={ }_{1}\right.$ in terms of distance constraint, we were able to obtain Lemm, and Lemm, through the distance constraint for the case where the distance is a special multiple ( $\beta=\sqrt{\hat{i}}$ ). Based on this result, we then obtained Theoren, determining the number of points that should be given for number of points with an inter-distance of less than , or for number of points with an inter-distance of or more to necessarily exist given arbitrary points on a plane.

Theorem 3 . For any two positive real numbers $\alpha, \beta$, and two natural numbers $x, y$, the following is true.

$$
\text { When } \quad m=\left[\log \sqrt{3}\left(\frac{\beta}{\alpha}\right)\right]=\left[2 \log _{3}\left(\begin{array}{l}
\beta \\
a
\end{array}\right) \quad T=\left\{\begin{array}{c}
3,7^{m}(m>0, \text { i.e } \beta> \\
3,(m \leq 0, \text { i. e } \beta \leq \alpha
\end{array}\right.\right.
$$

\section{, $\boldsymbol{K B}(\boldsymbol{\alpha}, \boldsymbol{\beta}, \boldsymbol{x}, \boldsymbol{y}) \leq$ TxyLemma 3}

By modifying Lemmawe obtained Lemmaand Lemma, and by refining Lemma for an optimal solution, we obtained Theorem as a better upper bound of $K B(\alpha, \beta, x$,

Theorem. For any two positive real numbers $\quad \alpha$,and two natural numbers $\quad x$, the following is true. 
If $m^{\prime}=\left[\log _{2 \cos \frac{\pi}{\alpha}\left(\frac{\beta}{\alpha}\right)}\right), T=\left\{\begin{array}{c}3, S^{m}\left(m^{\prime}>0, \text { i.e } \beta>\alpha\right) \\ 3,\left(m^{\prime} \leq 0, \text { i.e } \beta \leq \alpha\right)\end{array}, K B(\alpha, \beta, x, y) \leq\right.$ Txy

Subsequently, our results have proven to be practical in most cases compared with a self-evident result, i.e., a Ramsey number in a special case where $\alpha=\beta, m=$. However, in a special case for $\quad x$ (particularly when they are small), a Ramsey number was shown to be more accurate.

\subsection{Suggestions}

During this project, werealizedthat a creative solution to a common problem can be found when an idea is well developed. Although the ideas drawn from Lemma1 or Lemm are not necessarily easy, some mathematicians can still explore such ideas. By combining these two ideas, we easily obtainedthe upper bound of the minimum number of points that can be set for the generalized distance condition (i.e., upper bound of $K B(\alpha, \beta, x$, and we were further able to obtain a good upper bound. Possible improvement includes that the initial value was proven in a very specific case ( $r=1)$, resulting in broad results, which we have previously improved.Unfortunately, the Ramsey number is still more effective than the result we obtained, particularly when the $\quad x$ values are small, depending on the size of the subset (i.e., size of $\quad x$ ) to be obtained. This occurred because unlike the Ramsey number, we focused our conclusion, i.e., Theoremon the case where the size of the subset to be obtained, $\quad x$, is sufficiently large.

This study will provide various solutions to problems under various conditions or higher complexity by replacing distance with an angle or other distance values in combinatorial geometry in the future. Furthermore, this study will enable the estimation of maximum and minimum values when arranging limited itemsaccording to the distance condition; for example, in information communication networks as well as mathematics. This study can also be used in applied mathematics.

We did not consider the case where $\beta \leq$ when we refined the upper bound of $K B(\alpha, \beta, x$, In addition, $\left[\log _{r}\left(\frac{\beta}{\alpha}\right)=\log _{r}(\underset{\alpha}{\beta}\right.$ was used during the process of selecting the optimum value,which could result in errors when the value of $\quad \log _{r}$ (is small. Our future research will cover these issues.

\section{Reference}

[1] P. ERDOS, Graph theory and probability. II, Cunad. J. Mu\& 13 (1961), 346-352.

[2] J. SPENCER, Ramsey's Theorem - A New Lower Bound. JOURNAL OF COMBINATORIAL THEORY (A) 18 (1975), 108-115. 